International Journal of Modern Physics C Vol. 28, No. 12 (2017) 1799001 (13 pages) (C) World Scientific Publishing Company DOI: $10.1142 /$ S0129183117990017

\title{
Author Index (Volume 28)
}

Abdalla, A. S., see Eisa, M. H.

Achahbar, A., see Essouifi, M.

Ai, W.-H., see Liu, D.-W.

Alba-Cadena, A., see Arce-Guevara, V. E.

Alba, A., see León-Bejarano, $\mathrm{F}$.

Aliabadi, R., see Moradi, M.

Almeida, M. P., see Rodrigues, F. L. O.

Amirjanov, A., An evolution of cooperation in changing environment

Andrecut, M., Randomized kernel methods for least-squares support vector machines

Andrecut, M., Reservoir computing on the hypersphere

Andrecut, M., On the inherent competition between valid and spurious inductive inferences in Boolean data

Angco, M. J. G., see Paguirigan Jr., A. A.

Arce-Guevara, V. E., AlbaCadena, A. \& Mendez, M. O., Study of quadrature FIR filters for extraction of low-frequency instantaneous information in biophysical signals

Asaithambi, A., A series solution of the FalknerSkan equation using the crocco-wang transformation

Ashrafizaadeh, M., see Zadehgol, A.

$\mathbf{4}(2017) 1750043$
$\mathbf{9}(2017) 1750114$
$\mathbf{4}(2017) 1750046$
$\mathbf{5}(2017) 1750065$
$\mathbf{9}(2017) 1750116$
$\mathbf{5}(2017) 1750068$
$\mathbf{8}(2017) 1750105$
$\mathbf{1}(2017) 1750002$

2 (2017) 1750015

7 (2017) 1750095

12 (2017) 1750146

7 (2017) 1750092

5 (2017) 1750065

11 (2017) 1750139

1 (2017) 1750009
Baba, A. O., Bamaarouf, O., Rachadi, A. \& EzZahraouy, H., Effect of connection on transport between scale free networks

Bai, Y., Liu, S. \& Zhang, Z., Effective hybrid linkadding strategy to enhance network transport efficiency for scale-free networks

Balash, V. A., see Sidorov, S. P.

Bamaarouf, O., see Baba, A. O.

Bantang, J. Y., see Paguirigan Jr., A. A.

Ben Haddou, N., Ez-Zahraouy, H. \& Rachadi, A., Limited static and dynamic delivering capacity allocations in scale-free networks

Benyoussef, M., Ez-Zahraouy, H. \& Benyoussef, A., Optimal topology to minimizing congestion in connected communication complex network

Benyoussef, A., see Benyoussef, $\mathrm{M}$.

Bhattacharjee, K., Paul, D. \& Das, S., Pseudo-random number generation using $a$ 3-state cellular automaton Bonati, C., Coscetti, S., D'Elia, M., Mesiti, M., Negro, F., Calore, E., Schifano, S. F., Silvi, G. \& Tripiccione, R., Design and optimization of a portable LQCD Monte Carlo code using OpenACC
5 (2017) 1750064

8 (2017) 1750107

8 (2017) 1750103

5 (2017) 1750064

7 (2017) 1750092

11 (2017) 1750140

6 (2017) 1750073

6 (2017) 1750073

6 (2017) 1750078

5 (2017) 1750063 
Borzì, A., see Kułakowski, $\mathrm{K}$.

Bose, I., Pal, M. \& Karmakar, C., Allee dynamics: Growth, extinction and range expansion

Brum, R. M. \& Crokidakis, N., Dynamics of tax evasion through an epidemic-like model

Brutovsky, B., see Horvath, D.

Burini, D., De Lillo, S. \& Fioriti, G., Influence of drivers ability in a discrete vehicular traffic model

Cabrera-Trujillo, J. M., see Galván, C. G.

Cai, L., see Wang, J.

Cai, X., see Zhu, Y.

Calore, E., see Bonati, C.

Campos-Cantón, E., see Escalante-González, R. J.

Cao, C., see Liu, Y.

Carvente, O., see PerazaMues, G. G.

Chai, X., Gan, Z., Lu, Y., Chen, Y. \& Han, D., A novel image encryption algorithm based on the chaotic system and DNA computing

Chen, D.-B., see Sun, H.-L.

Chen, F., see Guan, J.

Chen, H., see Zong, X.

Chen, J., see Chen, L.

Chen, J., see Fu, Z.

Chen, L., Cui, H. \& Wang, L., Modified ghost fluid method on LBM with reduced spurious pressure oscillations for moving boundaries

Chen, L., Fang, H. \& Xie, Q., DFT study of the structural transformations and absorption properties of crystalline 2,6-dimethyl4-(diphenylmethylene)2,5-cyclohexadienone under hydrostatic compression
11 (2017) 1750133 $\mid \begin{aligned} & \text { Chen, L., Qu, J., Tao, Z., } \\ & \text { Xie, Q., Xie, G. \& Chen, } \\ & \text { J., First-principles study } \\ & \text { of the high-pressure beha- } \\ & \text { vior of crystalline benzoic } \\ & \text { acid (2017) } 1750074\end{aligned}$

Chen, R., see Qiu, R.

Chen, S., see Xiang, J.

Chen, T., Wu, Z.-H. \&

2 (2017) $1750023 \quad$ Wang, L., Donating money is not the only way to sustain cooperation in public goods game

Chen, W., Gao, Q. \& Xiong, H.-G., Uncover3 (2017) $1750030 \quad$ ing urban mobility patterns and impact of spatial distribution of places on movements

4 (2017) 1750048

3 (2017) 1750041

8 (2017) 1750109

5 (2017) 1750063

1 (2017) 1750008

10 (2017) 1750126

2 (2017) 1750021

5 (2017) 1750069

9 (2017) 1750112

6 (2017) 1750081

12 (2017) 1750142

10 (2017) 1750125

5 (2017) 1750059

4 (2017) 1750056

Chen, X. \& Lu, Z.-M., A real-time method to predict social media popularity

Chen, Y., see Chai, X.

Chen, Y.-G., see Xiao, W.

Cheng, G., see Ma, Y.

Cheng, J., see Shan, F.

Ch'ng, E., see Sun, H.-L.

Chong, W., see Peng, L.

Chopard, B., see Leclaire, S.

Corso, G., see dos Santos, M. C. V.

Coscetti, S., see Bonati, C.

Costa Filho, R. N., see Rodrigues, F. L. O.

Couder-Castañeda, C., see Orozco-Del-Castillo, M. G.

Crokidakis, N., see Lima, F. W. S.

Crokidakis, N., see Brum, R. M.

Cui, H., see Chen, L.

da Cunha Lima, A. T., see Santos, F. R.

da Cunha Lima, A. T., da Cunha Lima, I. C. \& de Almeida, M. P., Analysis of turbulence power spectra and velocity correlations in a pipeline with obstructions

da Cunha Lima, I. C., see da Cunha Lima, A. T.

2 (2017) $1750027 \quad$ Santos, F. R. $6(2017) 1750079$
10 (2017) 1750125

4 (2017) 1750045

6 (2017) 1750084

12 (2017) 1750149

1 (2017) 1750004

12 (2017) 1750144

5 (2017) 1750069

8 (2017) 1750106

6 (2017) 1750082

3 (2017) 1750038

9 (2017) 1750112

3 (2017) 1750033

7 (2017) 1750085

6 (2017) 1750076

5 (2017) 1750063

8 (2017) 1750105

6 (2017) 1750075

10 (2017) 1750123

2 (2017) 1750023

4 (2017) 1750056

6 (2017) 1750079

2 (2017) 1750019

2 (2017) 1750019 
da Silva Costa, G., see Santos, F. R.

da Silva, L. D., dos Santos, J. L. L., Ranciaro Neto, A., Sales, M. O. \& de Moura, F. A. B. F., Oneelectron propagation in Fermi, Pasta, Ulam disordered chains with Gaussian acoustic pulse pumping

Darabiha, N., see Hosseini, S. A.

Das, S., see Bhattacharjee, $\mathrm{K}$.

de Almeida, M. P., see da Cunha Lima, A. T.

de Almeida, M. P., see Santos, F. R.

De Lillo, S., see Burini, D.

De Lillo, S., Fioriti, G. \& Prioriello, M. L., On the modeling of epidemics under the influence of risk perception

de Moura, F. A. B. F., see da Silva, L. D.

D'Elia, M., see Bonati, C.

Deng, M. \& Feng, Y. Higher order two-scale finite element error analysis for thermoelastic problem in quasi-periodic perforated structure

Deng, Y., see Huang, Z. G.

Dereli, Y., see Kaplan, A. G.

Ding, C., Yao, H., Du, J., Peng, X., Wang, Z. \& Zhao, J., Cascading failure in interconnected weighted networks based on the state of link

Ding, Y., see Guo, Y.

Ding, Y., see Guo, Y.

Dong, Z., Fang, Y., Tian, M. \& Kong, Z., The influence of the depth of $k$-core layers on the robustness of interdependent networks against cascading failures

Dong, L.-Y., see Jin, C.-J.

Dorantes-Méndez, G., see León-Bejarano, F.
6 (2017) 1750079

8 (2017) 1750100

12 (2017) 1750141

6 (2017) 1750078

2 (2017) 1750019

6 (2017) 1750079

3 (2017) 1750030

4 (2017) 1750051

8 (2017) 1750100

5 (2017) 1750063

7 (2017) 1750097

6 (2017) 1750071

1 (2017) 1750011

3 (2017) 1750040

12 (2017) 1750147

6 (2017) 1750072

2 (2017) 1750020

2 (2017) 1750016

9 (2017) 1750116 dos Santos Lima, G. Z., see dos Santos, M. C. V. dos Santos, J. L. L., see da Silva, L. D.

dos Santos, M. C. V., Macedo-Filho, A., dos Santos Lima, G. Z. \& Corso, G., Self-organized energetic model for collective activity on animal tissue

Dybiec, B., Fighting for resources: Two leaders in the money addicted social hierarchies

Echab, H. \& Ez-Zahraouy, H., Dynamic characteristics of traffic flow with consideration of crossing pedestrians' behavior at a nonsignalized T-shaped intersection

Echab, H., see Khallouk, A.

Echab, H., Marzoug, R., Lakouari, N. \& Ez-Zahraouy, H., Studies of vehicle overtaking dynamics and its influence on traffic flow at a bidirectional road

Eisa, M. H. \& Abdalla, A. S., Dynamics of supersolid crystals in microcavity polariton condensates

Erkol, Ş. \& Yücel, G., Influence maximization based on partial network structure information: $A$ comparative analysis on seed selection heuristics

Escalante-González, R. J. \& Campos-Cantón, E., Generation of chaotic attractors without equilibria via piecewise linear systems

Eshghinejadfard, A., see Hosseini, S. A.

Essouifi, M. \& Achahbar, A., A mixed SIR-SIS model to contain a virus spreading through networks with two degrees
6 (2017) 1750076

3 (2017) 1750040

1 (2017) 1750010

11 (2017) 1750134

7 (2017) 1750093

6 (2017) 1750077

6 (2017) 1750076

8 (2017) 1750100

4 (2017) 1750043

10 (2017) 1750122

1 (2017) 1750008

12 (2017) 1750141

9 (2017) 1750114 
Ez-Zahraouy, H., see Khallouk, A.

Ez-Zahraouy, H., see Ben Haddou, N.

Ez-Zahraouy, H., see Baba, A. O.

Ez-Zahraouy, H., see Echab, H.

Ez-Zahraouy, H., see Benyoussef, $M$.

Ez-Zahraouy, H., see Echab, H.

Faizliev, A. R., see Sidorov, S. P.

Fang, H., see Chen, L.

Fang, J.-Q., see Liu, Q.

Fang, Y., see Dong, Z.

Fan, C., Jin, Y., Huo, L.A., Liu, C. \& Yang, Y., Epidemic spreading of interacting diseases with activity of nodes reshapes the critical threshold

Farghadani, O., see Omidvar, P.

Feng, Y., see Deng, M.

Feng, Z., see Liu, Y.

Feng, Z., see Guan, J.

Fenner, T., Kaufmann, E., Levene, M. \& Loizou, G., A multiplicative process for generating a beta-like survival function with application to the UK 2016 EU referendum results

Fernández-Anaya, G., see Rosas-Jaimes, O. A.

Filho, R. N. C., see Freire, M. M.

Fioriti, G., see Burini, D.

Fioriti, G., see De Lillo, S.

Freire, M. M., Filho, R. N. C. \& Leite, R. V., Green's functions theory for an interstitial magnetic impurity in a transverse Ising ferromagnet

$\mathrm{Fu}$, J. \& Wu, J., Reinterpretaion of the friendship paradox

\section{7(2017) 1750093 \\ 11 (2017) 1750140 \\ 5 (2017) 1750064 Yang, L. \& Chen, J., Simulation study of over- taking in pedestrian flow using floor field cellular automaton model}

11 (2017) 1750134

6 (2017) 1750073

6 (2017) 1750077

8 (2017) 1750103

2 (2017) 1750027

5 (2017) 1750057

2 (2017) 1750020

1 (2017) 1750013

10 (2017) 1750119

7 (2017) 1750097

10 (2017) 1750126

6 (2017) 1750081

11 (2017) 1750132

10 (2017) 1750130

4 (2017) 1750049

3 (2017) 1750030

4 (2017) 1750051

4 (2017) 1750049

2 (2017) 1750024
Fu, Z., Xia, L., Yang, H., Liu, X., Ma, J., Luo, L.,

$\mathrm{Fu}, \mathrm{Z}$., see Zhu, K.

Gadella, M., Lara, L. P. \& Negro, J., A qualitative study of a nanotube model using an iterative Taylor method

Gallegos, A., see MedinaGuevara, M. G.

Galván, C. G., CabreraTrujillo, J. M., Hernández-Hernández, I. J. \& Pérez, L. A., Molecular dynamics approach for crystal structures of methane $A$ and $B$

Gang, R., see Zhengfeng, $\mathrm{H}$.

Gan, Z., see Chai, X.

Gao, Q., see Chen, W.

Garibaldi, J. M., see Sun, H.-L.

Gazda, J., see Horvath, D.

Geroyannis, V. S., Tzelati, E. E. \& Karageorgopoulos, V. G., Nonradial oscillations of neutron stars and emitted gravitational waves: Computing strongly damped normal modes

Ghotbabadi, B. B., see Moradi, M.

Giedt, J., see Howarth, D.

Gong, J., see Li, Y.

Görlich, A., Kalomenopoulos, M., Savvidy, K. \& Savvidy, G., Distribution of periodic trajectories of $C-K$ systems MIXMAX pseudorandom number generator

Gronek, P., see Kułakowski, K.
5 (2017) 1750059

3 (2017) 1750034

3 (2017) 1750036

5 (2017) 1750058

4 (2017) 1750048

10 (2017) 1750121

5 (2017) 1750069

1 (2017) 1750004

9 (2017) 1750112

5 (2017) 1750062

6 (2017) 1750080

5 (2017) 1750068

10 (2017) 1750124

10 (2017) 1750128

3 (2017) 1750032

11 (2017) 1750133 
Author Index

Guan, J., Wang, K., Chen, F. \& Feng, Z., Evolution of pedestrian evacuation considering different human behaviors

Guo, L. M., Zhu, H. B. \& Zhang, N. X., Numerical study of traffic flow considering the probability density distribution of the traffic density

Guo, L.-J., see Xiang, J.

Guo, Q., see Yu, P.

Guo, W., see Wang, T.

Guo, X., see Shan, F.

Guo, X., see Kong, D.

Guo, Y., Ding, Y., Liu, Q. \& Zhao, X., DFT study of the structural and electronic properties of crystalline 2-benzylidene-1indanone under different hydrostatic pressures

Guo, Y., Ding, Y., Zhao, X. \& Liu, Q., Structural and electronic properties transitions induced by different pressures in crystalline nalidixic acid

Guo, Z. N., see Huang, Z. $\mathrm{G}$.

Hamioud, F., see Mubarak, A. A.

Han, D., see Chai, X.

Han, J.-T., see $\mathrm{Yu}, \mathrm{P}$.

Han, X., see Yang, Y.

Hao, R., see Wang, P.

Hao, R., see Huo, J.

He, J., see Wei, J.

He, M., see Pan, Q.

He, S., Yi, G. \& Wu, Z., Exponential synchronization in complex-variable network with distributed delays via intermittent control

He, Y., see Li, S.-B.

He, Z., see Yang, J.

Heidari, H., see Mohebbi, $\mathrm{R}$.

Hernández-Gómez, J. J., see Orozco-Del-Castillo, M. G.

Hernández-Hernández, I. J., see Galván, C. G.
6 (2017) 1750081

2 (2017) 1750026

6 (2017) 1750084

3 (2017) 1750029

12 (2017) 1750131

3 (2017) 1750038

6 (2017) 1750083

6 (2017) 1750072

12 (2017) 1750147

6 (2017) 1750071

12 (2017) 1750148

5 (2017) 1750069

3 (2017) 1750029

9 (2017) 1750115

11 (2017) 1750135

12 (2017) 1750150

12 (2017) 1750145

1 (2017) 1750003

7 (2017) 1750089

9 (2017) 1750117

7 (2017) 1750091

3 (2017) 1750042

6 (2017) 1750075

4 (2017) 1750048
Horvath, D., Gazda, J. \& Brutovsky, B., A new bio-inspired, populationlevel approach to the socioeconomic evolution of dynamic spectrum access services

Hosseini, A., Iranmanesh, M., Javaran, E. J. \& Zadehgol, A., Application of lattice kinetic models with Tsallis entropy in simulating fluid flow through porous media

Hosseini, S. A., Darabiha, N., Thévenin, D. \& Eshghinejadfard, A., Stability limits of the single relaxation-time advection-diffusion lattice Boltzmann scheme

Howarth, D. \& Giedt, J., The sigma meson from lattice $Q C D$ with two-pion interpolating operators

$\mathrm{Hu}, \mathrm{R}$., see Yang, F.

$\mathrm{Hu}, \mathrm{R}$., see Yao, Y.

$\mathrm{Hu}, \mathrm{R}$., see Yao, Y.

Huang, J.-W., see Li, S.-B.

Huang, Z. G., Yue, T. M., Deng, Y. \& Guo, Z. N., Extendable outflow boundary conditions for dissipative particle dynamics simulation

Huo, J., see Wang, P.

Huo, J., Wang, X.-M., Hao, R. \& Wang, P., Statistical dynamics of regional populations and economies

Huo, L.-A., see Fan, C.

Iranmanesh, M., see Hosseini, A.

Javaran, E. J., see Hosseini, A.

Jia, H., see Li, Y.

Jia, H., see Li, Y.

Jiang, P. \& Yan, X., Stability analysis and control models for rumor spreading in online social networks

Jiang, R., see Jin, C.-J.
5 (2017) 1750062

9 (2017) 1750110

12 (2017) 1750141

10 (2017) 1750124

1 (2017) 1750014

4 (2017) 1750053

8 (2017) 1750101

9 (2017) 1750117

6 (2017) 1750071

11 (2017) 1750135

12 (2017) 1750150

1 (2017) 1750013

9 (2017) 1750110

9 (2017) 1750110

10 (2017) 1750128

2 (2017) 1750025 
Jiang, Z.-Y. \& Ma, J.-F., Effects of packet retransmission with finite packet lifetime on traffic capacity in scale-free networks

Jiang, Z.-Y. \& Ma, J.-F., An efficient local cascade defense method in complex networks

Jian, Z., see Wang, L.

Jianping, L., see Yujun, Y. Jin, C.-J., Wang, W., Jiang, R. \& Dong, L.Y., Simulating pedestrian flow by an improved twoprocess cellular automaton model

Jin, N., see Wang, H.

Jin, Y., see Fan, C.

Jing, W., see Ya-Qi, W.

Juan, W. X., see Yong, Z.

Juan, W. X., Ze, G. S., Lei, J. \& Mo, C., Cascading failures mechanism based on betweennessdegree ratio distribution with different connecting preferences

Kalomenopoulos, M., see Görlich, A.

Kaplan, A. G. \& Dereli, Y., Numerical solutions of the GEW equation using MLS collocation method

Karageorgopoulos, V. G., see Geroyannis, V. S.

Karmakar, C., see Bose, I.

Katırcıoğlu, Ş., see Mohammad, R.

Kaufmann, E., see Fenner, T.

Kaupužs, J., Melnik, R. V. N. \& Rimšāns, J., Corrections to finite-size scaling in the $3 D$ Ising model based on nonperturbative approaches and Monte Carlo simulations

Khallouk, A., Lakouari, N., Echab, H. \& EzZahraouy, H., Traffic flow behavior at a single lane traffic circle

Khmou, Y., A case study in bifurcation theory
5 (2017) 1750066

3 (2017) 1750031

7 (2017) 1750098

2 (2017) 1750028

2 (2017) 1750016

1 (2017) 1750007

1 (2017) 1750013

5 (2017) 1750060

4 (2017) 1750050

4 (2017) 1750052

3 (2017) 1750032

1 (2017) 1750011

6 (2017) 1750080

6 (2017) 1750074

3 (2017) 1750039

11 (2017) 1750132

4 (2017) 1750044

7 (2017) 1750093

8 (2017) 1750104
Khène, S., see Tag, M. A.

Klimenko, S., Nikitin, I., Nikitina, L., Konich, K. \& Reinartz, K., Numeri-

9 (2017) 1750113 cal modeling of relativistic networks

3 (2017) 1750035

Klimenko, S., Nikitin, I. \& Nikitina, L., Numerical solutions of Einstein field equations with radial dark matter

7 (2017) 1750096

Kong, D., Guo, X. \& Wu, D., The influence of heavy vehicles on traffic dynamics around onramp system: Cellular automata approach

Kong, Z., see Dong, Z.

Konich, K., see Klimenko, $\mathrm{S}$.

Kułakowski, K., Gronek, P. \& Borzì, A., Paradox of integration - mean field approach

11 (2017) 1750133

Kwa, K. H., see Mohd Ali, Y. E.

Lakouari, N., see Echab, H.

Lakouari, N., see Khallouk, A.

Lara, L. P., see Gadella, M.

Latt, J., see Leclaire, S.

Lazo, M. V., see Shinbrot, T.

Leclaire, S., Parmigiani, A., Chopard, B. \& Latt, J., Three-dimensional lattice Boltzmann method benchmarks between color-gradient and pseudo-potential immiscible multi-component models

Lee, Y. H., see Yevick, D.

Lei, J., see Yong, Z.

Lei, J., see Juan, W. X.

Leite, R. V., see Freire, M. $\mathrm{M}$.

6 (2017) 1750083

2 (2017) 1750020

3 (2017) 1750035

9 (2017) 1750118

6 (2017) 1750077

7 (2017) 1750093

3 (2017) 1750036

7 (2017) 1750085

2 (2017) 1750018

7 (2017) 1750085

1 (2017) 1750012

4 (2017) 1750050

4 (2017) 1750052

4 (2017) 1750049

León-Bejarano, F., Ramírez-Elías, M., Mendez, M. O., Dorantes-Méndez, G., Rodríguez-Aranda, Ma. del C. \& Alba, A., Denoising of Raman spectroscopy for biological samples based on empirical mode decomposition

Levene, M., see Fenner, T. 
Li, H.-J., see Xiang, J.

Li, H., see Yang, J.

Li, J.-M., see Xiang, J.

Li, J., see Li, Y.

Li, K., Ma, X. \& Shao, F., An improved network model for railway traffic

Li, K., see Yang, F.

Li, M., see Yang, F.

Li, R.-D., see Yu, P.

Li, S.-B., He, Y., Liu, J.H., Zhang, Z.-G. \& Huang, J.-W., Congestion control strategy on complex network with privilege traffic

Li, W., see Zhu, Y.

Li, Y., Jia, H., Li, J., Gong, J. \& Sun, K., Pedestrian evacuation behavior analysis and simulation in multi-exits case

Li, Y., Jia, H., Zhou, Y.N. \& Yang, L., Simulation research on pedestrian counter flow subconscious behavior

Li, Y., see Liu, Q.

Li, Y., see Pan, G.

Liang, X., see Ma, Y.

Liang, Y., see Wang, J.

Liao, H., see Yang, J.

Lima, F. W. S., see Sumour, M. A.

Lima, F. W. S., Equilibrium and nonequilibrium models on solomon networks with two square lattices

Lima, F. W. S. \& Crokidakis, N., Nonequilibrium opinion dynamics on triangular, honeycomb, and Kagome lattices

Liu, C.-C., see Xiang, J.

Liu, C., see Fan, C.

Liu, D.-W., Shi, Z.-K. \& Ai, W.-H., An improved car-following model from the perspective of driver's forecast behavior

liu, J.-G., see Yu, P.

Liu, J.-H., see Li, S.-B.
6 (2017) 1750084

7 (2017) 1750091

6 (2017) 1750084

10 (2017) 1750128

3 (2017) 1750037

1 (2017) 1750014

1 (2017) 1750014

3 (2017) 1750029

9 (2017) 1750117

8 (2017) 1750109

10 (2017) 1750128

2 (2017) 1750025

5 (2017) 1750057

7 (2017) 1750090

6 (2017) 1750082

10 (2017) 1750127

7 (2017) 1750091

2 (2017) 1750022

8 (2017) 1750099

10 (2017) 1750123

6 (2017) 1750084

1 (2017) 1750013

4 (2017) 1750046

3 (2017) 1750029

9 (2017) 1750117
Liu, L., see Ye, F.

11 (2017) 1750129

12 (2017) 1750147

6 (2017) 1750072

Liu, Q., see Guo, Y.

Liu, Q., Fang, J.-Q. \& Li, Y., Some characteristics of supernetworks based on unified hybrid network theory framework

Liu, S., see Bai, Y.

Liu, X., see Fu, Z.

Liu, Y., see Wang, T.

Liu, Y., Cao, C., Zhou, Y. \& Feng, Z., A real-time control method-based simulation for high-speed trains on large-scale rail network

Liu, Z., see Ma, Y.

Loizou, G., see Fenner, T.

Lou, Q., Zang, C., Yang, M. \& Xu, H., Lattice Boltzmann simulation of immiscible displacement in the cavity with different channel configurations

Lu, Y., see Chai, X.

Lu, Z.-M., see Chen, X.

Luo, L., see Fu, Z.

Lv, F., see Wang, Y.

Ma, J.-F., see Jiang, Z.-Y.

Ma, J.-F., see Jiang, Z.-Y.

$\mathrm{Ma}$, J., see $\mathrm{Fu}, \mathrm{Z}$.

Ma, J., see Yang, Y.

Ma, J., see Yang, Y.

Ma, T., see Wang, J.

Ma, X., see Li, K.

Ma, Y., Cheng, G., Liu, Z. \& Liang, X., Clusteringbased link prediction in scientific coauthorship networks

Macías-Díaz, J. E., see Medina-Guevara, M. G.

Macedo-Filho, A., see dos Santos, M. C. V.

Mao, Y. F., see Peng, Y.

Marzoug, R., see Echab, H.

Mateus, P., see Rodrigues, J.

Maziero, J., Computing partial traces and reduced density matrices
10 (2017) 1750126

6 (2017) 1750082

11 (2017) 1750132

11 (2017) 1750136

5 (2017) 1750069

12 (2017) 1750144

5 (2017) 1750059

9 (2017) 1750111

3 (2017) 1750031

5 (2017) 1750066

5 (2017) 1750059

7 (2017) 1750087

9 (2017) 1750115

10 (2017) 1750127

3 (2017) 1750037

6 (2017) 1750082

5 (2017) 1750058

6 (2017) 1750076

9 (2017) 1750120

6 (2017) 1750077

4 (2017) 1750055

1 (2017) 1750005 
Medina-Guevara, M. G., Macías-Díaz, J. E., Gallegos, A. \& VargasRodríguez, H., Consensus formation simulation in a social network modeling controversial opinion dynamics with pairwise interactions

Melnik, R. V. N., see Kaupužs, J.

Mendez, M. O., see ArceGuevara, V. E.

Mendez, M. O., see LeónBejarano, F.

Meng, Q., see Zhang, X.

Mesiti, M., see Bonati, C.

Mo, C., see Juan, W. X.

Mohammad, R. \& Katırcroğlu, Ş., First-principles calculations for mechanical and electronic features of strained $\mathrm{GaP}$ nanowires

Mohd Ali, Y. E., Kwa, K. H. \& Ratnavelu, K., Predicting new drug indications from network analysis

Mohebbi, R. \& Heidari, H., Lattice Boltzmann simulation of fluid flow and heat transfer in a parallel-plate channel with transverse rectangular cavities

Morad, M. R., see Pourabdian, M.

Moradi, M., Ghotbabadi, B. B. \& Aliabadi, R., The study of uniaxial-biaxial phase transition of confined hard ellipsoids using density functional theory

Moukarzel, C. F., see Peraza-Mues, G. G.

Mozaffari, M. S. \& Roohi, E., On the thermallydriven gas flow through divergent micro/nanochannels

Mubarak, A. A. \& Hamioud, F., Hydrogen storage in the TiCo and TiNi alloys

\section{5 (2017) 1750058 \\ 4 (2017) 1750044 \\ 5 (2017) 1750065 \\ 9 (2017) 1750116 \\ 11 (2017) 1750137 \\ 5 (2017) 1750063 \\ 4 (2017) 1750052}

3 (2017) 1750039

9 (2017) 1750118

3 (2017) 1750042

4 (2017) 1750054

\section{5 (2017) 1750068}

2 (2017) 1750021

12 (2017) 1750143

12 (2017) 1750148
Murguía, J. S., Scaling analysis of hyperchaotic time series

Negro, F., see Bonati, C.

Negro, J., see Gadella, M.

$\mathrm{Ni}$, C., see Wang, J.

Nikeghbali, P., see Omidvar, $\mathrm{P}$.

Nikitin, I., see Klimenko, S.

Nikitina, L., see Klimenko, $\mathrm{S}$.

Nikitina, L., see Klimenko, S.

Nikitin, I., see Klimenko, S.

Ning, W., see Wang, L.

Niu, Y., see Zhu, K.

Omidvar, P., see Pourabdian, $\mathbf{M}$.

Omidvar, P., Farghadani, O. \& Nikeghbali, P., $\mathrm{SPH}$ for impact force and ricochet behavior of water-entry bodies

Orozco-Del-Castillo, M. G., Ortiz-Alemán, J. C., Couder-Castañeda, C., Hernández-Gómez, J. J. \& Solís-Santomé, A., High solar activity predictions through an artificial neural network

Ortiz-Alemán, J. C., see Orozco-Del-Castillo, M. G.

Paguirigan Jr., A. A., Angco, M. J. G. \& Bantang, J. Y., A fragmentation model of earthquakelike behavior in internet access activity

Pal, M., see Bose, I.

Pan, G., Zhang, Y., Li, Y., Zhang, C., Zhao, Z. \& Zeng, Z., Orientationdependent behaviors of $H$ dissolution and diffusion near $W$ surfaces: $A$ first-principles study

Pan, Q., Qin, Y., Xu, Y., Tong, M. \& He, M., Opinion evolution in open community

Parmigiani, A., see Leclaire, S.
7 (2017) 1750094

5 (2017) 1750063

3 (2017) 1750036

3 (2017) 1750041

10 (2017) 1750119

7 (2017) 1750096

7 (2017) 1750096

3 (2017) 1750035

3 (2017) 1750035

7 (2017) 1750098

3 (2017) 1750034

4 (2017) 1750054

10 (2017) 1750119

6 (2017) 1750075

6 (2017) 1750075

7 (2017) 1750092

6 (2017) 1750074

7 (2017) 1750090

1 (2017) 1750003

7 (2017) 1750085 
Author Index

Paul, D., see Bhattacharjee, K.

Paunković, N., see Rodrigues, J.

Peng, Y., Mao, Y. F., Wang, B. \& Xie, B. Study on $C-S$ and $P-R$ EOS in pseudo-potential lattice Boltzmann model for two-phase flows

Peng, L., Chong, W. \& Yongli, L., Link prediction measures considering different neighbors effects and application in social networks

Peng, X., see Ding, C.

Pengjun, Z., see Zhengfeng, $\mathrm{H}$

Peraza-Mues, G. G., Carvente, O. \& Moukarzel, C. F., Rotation in a gravitational billiard

Pourabdian, M., Omidvar, P. \& Morad, M. R., Multiphase simulation of liquid jet breakup using smoothed particle hydrodynamics

Pérez, L. A., see Galván, C. G.

Prioriello, M. L., see De Lillo, S.

Qi, Z., see Yang, Y.

Qin, J. \& Zheng, M., New York city taxi trips: Dynamic networks following inconsistent power law Qin, X., see Wang, X.

Qin, Y., see Pan, Q.

Qiu, R., Chen, R. \& You, Y., An implicit-explicit finite-difference lattice Boltzmann subgridmethod on nonuniform meshes

Qiu, Y., see Yao, Y.

Quezada-Téllez, L. A., see Rosas-Jaimes, O. A.

Qu, J., see Chen, L.

Rachadi, A., see Baba, A. O

Rachadi, A., see Ben Haddou, N.

Ramírez-Elías, M., see León-Bejarano, F.
Ranciaro Neto, A., see da Silva, L. D.

Ratnavelu, K., see Mohd

4 (2017) $1750055 \quad$ Ali, Y. E. 9 (2017) 1750118

Ratnavelu, K., see Tan, M. S. A.

Reinartz, K., see Klimenko, $\mathrm{S}$. J.

Rodríguez-Aranda, Ma. del C., see León-Bejarano, $\mathrm{F}$.

Rodrigues, F. L. O., Almeida, M. P. \& Costa Filho, R. N., Phase field model for dendritic growth with impurities

10 (2017) $1750121 \quad$ Rodrigues, J., Paunković, N. \& Mateus, P., A simulator for discrete quantum walks on lattices 2 (2017) 1750021 Roohi, E., see Mozaffari, M. S.

Rosas-Jaimes, O. A., Quezada-Téllez, L. A. \& Fernández-Anaya, G., Stability analysis in Greenberg's traffic model

Sales, M. O., see da Silva, L. D.

4 (2017) 1750048

4 (2017) 1750051

7 (2017) 1750087

7 (2017) 1750088

1 (2017) 1750006

1 (2017) 1750003

4 (2017) 1750045

8 (2017) 1750101

10 (2017) 1750130

10 (2017) 1750125

5 (2017) 1750064

11 (2017) 1750140

9 (2017) 1750116
Santos, F. R., da Silva Costa, G., da Cunha Lima, A. T., de Almeida, M. P. \& da Cunha Lima, I. C., Detection of memory loss of symmetry in the blockage of a turbulent flow within a duct

Savvidy, G., see Görlich, A.

Savvidy, K., see Görlich, A.

Schifano, S. F., see Bonati, C.

See, S., see Sun, H.-L.

Shan, F., Guo, X., Tu, J., Cheng, J. \& Zhang, D., Multi-relaxation-time lattice Boltzmann modeling of the acoustic field generated by focused transducer Shao, F., see Li, K.
8 (2017) 1750100

9 (2017) 1750118

2 (2017) 1750017

3 (2017) 1750035

4 (2017) 1750044

9 (2017) 1750116

8 (2017) 1750105

4 (2017) 1750055

12 (2017) 1750143

10 (2017) 1750130

8 (2017) 1750100

6 (2017) 1750079

3 (2017) 1750032

3 (2017) 1750032

5 (2017) 1750063

9 (2017) 1750112

3 (2017) 1750038

3 (2017) 1750037 
Shi, Q., see Zhu, K.

Shi, Z.-K., see Liu, D.-W.

Shi, Z.-K., see Zhou, J.

Shinbrot, T., Lazo, M. V.

\& Siu, T., Network simulations of optical illusions

Sidorov, S. P., Faizliev, A. R. \& Balash, V. A., Measuring long-range correlations in news flow intensity time series

Silvi, G., see Bonati, C.

Siu, T., see Shinbrot, T.

Solís-Santomé, A., see Orozco-Del-Castillo, M. G.

Song, J., see Wang, P.

Su, M. L., see Wang, Y.

Sumour, M. A. \& Lima, F. W. S., A systematic investigation of the gap in various Barabási-Albert and Erdös-Rényi networks

Sun, H.-L., Ch'ng, E., Yong, X., Garibaldi, J. M., See, S. \& Chen, D.B., An improved gametheoretic approach to uncover overlapping communities

Sun, K., see Li, Y.

Sun, Q., see Yao, Y.

Tag, M. A. \& Khène, S., Automatic generation of essentially distinct Hugenholtz diagrams

Tan, M. S. A., Ujum, E. A. \& Ratnavelu, K., Social network analysis of character interaction in the Stargate and Star Trek television series

Tao, Z., see Chen, L.

Thévenin, D., see Hosseini, S. A

Tian, M., see Dong, Z.

Tong, M., see Pan, Q.

Torreão, J. R. A., Generalized signal-tuned Gabor approach for signal representation and analysis

Tripiccione, R., see Bonati, C.

Tu, J., see Shan, F.
3 (2017) 1750034

4 (2017) 1750046

7 (2017) 1750086

2 (2017) 1750018

8 (2017) 1750103

5 (2017) 1750063

2 (2017) 1750018

6 (2017) 1750075

11 (2017) 1750135

9 (2017) 1750111

2 (2017) 1750022

9 (2017) 1750112

10 (2017) 1750128

8 (2017) 1750101

9 (2017) 1750113

2 (2017) 1750017

10 (2017) 1750125

12 (2017) 1750141

2 (2017) 1750020

1 (2017) 1750003

1 (2017) 1750001

5 (2017) 1750063

3 (2017) 1750038
Tzelati, E. E., see Geroyannis, V. S.

6 (2017) 1750080

Ujum, E. A., see Tan, M. S. A.

2 (2017) 1750017

Vargas-Rodríguez, H., see Medina-Guevara, M. G.

Voulgarakis, N. K., $A$ stochastic reaction-diffusion model for protein aggregation on DNA

Wang, X.-M., see Wang, P.

Wang, X. \& Qin, X., Detecting communities by asymmetric intimacy in directed-weighted network

Wang, B., see Peng, Y.

Wang, B.-H., see Ye, F.

Wang, C., see Zong, X.

Wang, C., see Zhang, C.

Wang, C.-P., see Zhou, J.

Wang, H., Zhai, L., Jin, N. \& Wang, Y., Multiscale symbolic time reverse analysis of gas-liquid two-phase flow structures

Wang, J., see Wang, M.

Wang, J., Wang, X., Cai, L., Ni, C., Xie, W. \& $\mathrm{Xu}, \mathrm{B} .$, Universal resilience patterns in cascading load model: More capacity is not always better

Wang, J., Zeng, Y., Wang, P., Liang, Y. \& Ma, T., The evolution of pairing correlation in the anisotropic triangular lattice

Wang, K., see Guan, J.

Wang, L., see Yang, X.

Wang, L., see Chen, $T$.

Wang, L., see Chen, L.

Wang, L., Jian, Z. \& Ning, W., Phonon, elastic and thermodynamic properties of $\mathrm{Ll}_{2}$ phase $\mathrm{Rh}_{3} \mathrm{Zr}$ under pressure from firstprinciples

Wang, M. \& Wang, J. Multiscale volatility duration characteristics on financial multi-continuum percolation dynamics

Wang, P., see Wang, J.
5 (2017) 1750058

8 (2017) 1750102

11 (2017) 1750135

1 (2017) 1750006

9 (2017) 1750120

11 (2017) 1750129

12 (2017) 1750142

8 (2017) 1750108

7 (2017) 1750086

1 (2017) 1750007

5 (2017) 1750067

3 (2017) 1750041

10 (2017) 1750127

6 (2017) 1750081

11 (2017) 1750138

12 (2017) 1750149

4 (2017) 1750056

7 (2017) 1750098

5 (2017) 1750067

10 (2017) 1750127 
Wang, P., see Huo, J.

Wang, P., Song, J., Huo, J., Hao, R. \& Wang, X.M., Towards understanding what contributes to forming an opinion

Wang, Q. A., see Zhu, Y.

Wang, T., Liu, Y., Wen, W., Guo, W. \& Yin, Y., The phosphorene under the external electronic field and strain

Wang, W., see Jin, C.-J.

Wang, X., see Wei, J.

Wang, X., see Wang, J.

Wang, X., see Zhang, C.

Wang, X.-M., see Huo, J.

Wang, Y., see Wang, $\mathrm{H}$.

Wang, Y., Wu, D. J., Lv, F. \& Su, M. L., Exploring activity-driven network with biased walks

Wang, Z., see Ding, C.

Wang, Z.-Z., see Xiang, J.

Wei, J., Wu, Z., You, L., He, J., Zhang, H. \& Wang, X., Modeling and simulation of group formation and crowd dynamics with smoke effect

Wen, W., see Wang, T.

Wenjun, X., see Zhengfeng, $\mathrm{H}$.

Wu, Q.\& Zhu, W., Toward a generalized theory of epidemic awareness in social networks

Wu, D. J., see Wang, Y.

Wu, D., see Kong, D.

$\mathrm{Wu}$, J., see $\mathrm{Fu}, \mathrm{J}$.

Wu, Z.-H., see Chen, T.

Wu, Z., see Wei, J.

$\mathrm{Wu}, \mathrm{Z}$., see $\mathrm{He}, \mathrm{S}$.

Xia, L., see $\mathrm{Fu}, \mathrm{Z}$.

Xia, Z., see Zhang, C.

Xiang, J., Wang, Z.-Z., Li, H.-J., Zhang, Y., Chen, S., Liu, C.-C., Li, J.-M. \& Guo, L.-J., Comparing local modularity optimization for detecting communities in networks
12 (2017) 1750150

11 (2017) 1750135

8 (2017) 1750109

12 (2017) 1750131

2 (2017) 1750016

12 (2017) 1750145

3 (2017) 1750041

8 (2017) 1750108

12 (2017) 1750150

1 (2017) 1750007

9 (2017) 1750111

3 (2017) 1750040

6 (2017) 1750084

12 (2017) 1750145

12 (2017) 1750131

10 (2017) 1750121

5 (2017) 1750070

9 (2017) 1750111

6 (2017) 1750083

2 (2017) 1750024

12 (2017) 1750149

12 (2017) 1750145

7 (2017) 1750089

5 (2017) 1750059

8 (2017) 1750108

6 (2017) 1750084
Xiao, W., Chen, Y.-G., Yang, Y.-P. \& Yang, C., The impact of intelligent vehicles on a two-route system with a work zone

Xie, B., see Peng, Y.

Xie, G., see Chen, L.

Xie, Q., see Chen, L.

Xie, Q., see Chen, L.

Xie, W., see Wang, J.

Xie, W., see Yang, J.

Xiong, H.-G., see Chen, W.

$\mathrm{Xu}, \mathrm{B}$., see Wang, J.

$\mathrm{Xu}, \mathrm{H}$., see Lou, Q.

$\mathrm{Xu}, \mathrm{Y}$., see Pan, Q.

Yan, X., see Jiang, P.

Yang, C., see Xiao, W.

Yang, F., see Yao, Y.

Yang, F., see Yao, Y.

Yang, F., Zhang, R., Yang, Z., Hu, R., Li, M., Yuan, Y. \& Li, K., Identifying the most influential spreaders in complex networks by an Extended Local K-Shell Sum

Yang, H., see Fu, Z.

Yang, H., see Yang, J.

Yang, J., Li, H., Liao, H., He, Z., Yang, H. \& Xie, W., Localization of information on communication networks of an opensource online community

Yang, L., see Li, Y.

Yang, L., see Fu, $\mathrm{Z}$.

Yang, M., see Lou, Q.

Yang, X. \& Wang, L., Multiple-relaxation-time lattice Boltzmann study of the magnetic field effects on natural convection of non-Newtonian fluids

Yang, Y., see Fan, C.

Yang, Y., see Zhu, K.

Yang, Y., Zhao, H., Ma, J. \& Han, X., Socialaware data dissemination in opportunistic mobile social networks
8 (2017) 1750106

9 (2017) 1750120

10 (2017) 1750125

10 (2017) 1750125

2 (2017) 1750027

3 (2017) 1750041

7 (2017) 1750091

1 (2017) 1750004

3 (2017) 1750041

11 (2017) 1750136

1 (2017) 1750003

5 (2017) 1750061

8 (2017) 1750106

4 (2017) 1750053

8 (2017) 1750101

1 (2017) 1750014

5 (2017) 1750059

7 (2017) 1750091

7 (2017) 1750091

2 (2017) 1750025

5 (2017) 1750059

11 (2017) 1750136

11 (2017) 1750138

1 (2017) 1750013

3 (2017) 1750034

9 (2017) 1750115 
Yang, Y., Zhao, H., Ma, J., Qi, Z. \& Zhao, Y., An optimal routing strategy on scale-free networks Yang, Y.-P., see Xiao, W. Yang, Z., see Yang, F.

Yao, H., see Ding, C.

Yao, Y., Zhang, R., Yang, F., Yuan, Y., Sun, Q., Qiu, Y. \& Hu, R., Link prediction via layer relevance of multiplex networks

Yao, Y., Zhang, R., Yang, F., Yuan, Y., Hu, R. \& Zhao, Z., Link prediction based on local weighted paths for complex networks

Ya-Qi, W. \& Jing, W., SIR rumor spreading model considering the effect of difference in nodes' identification capabilities

Ye, F., Zhang, L., Wang, B.-H., Liu, L. \& Zhang, X.-Y., Extended shortest path selection for package routing of complex networks

Yevick, D. \& Lee, Y. H., Accelerated rare event sampling: Refinement and Ising model analysis

Yi, G., see He, S.

Yimei, Y., see Yujun, Y.

Yin, Y., see Wang, T.

Yong, X., see Sun, H.-L.

Yong, Z., Lei, J. \& Juan, W. X., Research on cascading failure in multilayer network with different coupling preference

Yongli, L., see Peng, L.

You, L., see Wei, J.

You, Y., see Qiu, R.

Yu, P., Guo, Q., Li, R.-D., Han, J.-T. \& liu, J.-G., Roles of clustering properties for degree-mixing pattern networks

Yuan, Y., see Yang, F.

Yuan, Y., see Yao, Y.

Yuan, Y., see Yao, Y.

Yücel, G., see Erkol, Ş.

Yue, T. M., see Huang, Z. G.
7 (2017) 1750087

8 (2017) 1750106

1 (2017) 1750014

3 (2017) 1750040

8 (2017) 1750101

4 (2017) 1750053

5 (2017) 1750060

11 (2017) 1750129

1 (2017) 1750012

7 (2017) 1750089

2 (2017) 1750028

12 (2017) 1750131

9 (2017) 1750112

4 (2017) 1750050

3 (2017) 1750033

12 (2017) 1750145

4 (2017) 1750045

3 (2017) 1750029

1 (2017) 1750014

4 (2017) 1750053

8 (2017) 1750101

10 (2017) 1750122

6 (2017) 1750071
Yujun, Y., Jianping, L. \& Yimei, Y., Multiscale multifractal multiproperty analysis of financial time series based on Rényi entropy

Zadehgol, A., see Hosseini, A.

Zadehgol, A. \& Ashrafizaadeh, M., On the entropy variations and the Maxwell relations

Zang, C., see Lou, Q.

Ze, G. S., see Juan, W. X.

Zeng, M., see Zhang, X.

Zeng, Y., see Wang, J.

Zeng, Z., see Pan, G.

Zhai, L., see Wang, H.

Zhai, Q., Zheng, S. \& Zheng, L., A kinetic theory based thermal lattice Boltzmann equation model

Zhang, C., see Pan, G.

Zhang, C., Wang, X., Wang, C. \& Xia, Z., Outer synchronization of complex networks with internal delay and coupling delay via aperiodically intermittent pinning control

Zhang, D., see Shan, F.

Zhang, H., see Wei, J.

Zhang, H.-L., see Zhou, J.

Zhang, L., see Ye, F.

Zhang, N. X., see Guo, L. M.

Zhang, R., see Yang, F.

Zhang, R., see Yao, Y.

Zhang, R., see Yao, Y.

Zhang, X., Zeng, M. \& Meng, Q., Asymmetric multiscale multifractal analysis of wind speed signals

Zhang, X.-Y., see Ye, F.

Zhang, Y., see Xiang, J.

Zhang, Y., see Pan, G.

Zhang, Z., see Bai, Y.

Zhang, Z.-G., see Li, S.-B.

Zhao, H., see Yang, Y.

Zhao, H., see Yang, Y.

Zhao, J., see Ding, C.

Zhao, X., see Guo, Y.
2 (2017) 1750028

9 (2017) 1750110

1 (2017) 1750009

11 (2017) 1750136

4 (2017) 1750052

11 (2017) 1750137

10 (2017) 1750127

7 (2017) 1750090

1 (2017) 1750007

4 (2017) 1750047

7 (2017) 1750090

8 (2017) 1750108

3 (2017) 1750038

12 (2017) 1750145

7 (2017) 1750086

11 (2017) 1750129

2 (2017) 1750026

1 (2017) 1750014

4 (2017) 1750053

8 (2017) 1750101

11 (2017) 1750137

11 (2017) 1750129

6 (2017) 1750084

7 (2017) 1750090

8 (2017) 1750107

9 (2017) 1750117

7 (2017) 1750087

9 (2017) 1750115

3 (2017) 1750040

12 (2017) 1750147 
Zhao, X., see Guo, Y.

Zhao, Y., see Yang, Y.

Zhao, Z., see Yao, Y.

Zhao, Z., see Pan, G.

Zhengfeng, H., Pengjun,

Z., Wenjun, X. \& Gang,

R., SAE for the prediction

of road traffic status from taxicab operating data and bus smart card data

Zheng, L., see Zhai, Q.

Zheng, M., see Qin, J.

Zheng, S., see Zhai, Q.

Zhou, J., Shi, Z.-K., Zhang, H.-L. \& Wang, C.-P., A new lattice model for single-lane traffic flow with the consideration of driver's memory during a period of time
6 (2017) 1750072

7 (2017) 1750087

4 (2017) 1750053

7 (2017) 1750090

10 (2017) 1750121

4 (2017) 1750047

7 (2017) 1750088

4 (2017) 1750047

7 (2017) 1750086
Zhou, Y., see Liu, Y.

10 (2017) 1750126

Zhou, Y.-N., see Li, Y.

2 (2017) 1750025

Zhu, H. B., see Guo, L. M.

Zhu, K., Yang, Y., Niu, Y., Fu, Z. \& Shi, Q., Modeling pedestrian flow on multi-storey stairs considering turning behavior Zhu, W., see Wu, Q.

Zhu, Y., Wang, Q. A., Li, W. \& Cai, X., Uncertainty and sensitivity analysis to complex systems

Zong, X., Wang, C. \& Chen, H., An evacuation model based on co-evolutionary multi-particle swarms optimization for pedestrian-vehicle mixed traffic flow
3 (2017) 1750034

5 (2017) 1750070

2 (2017) 1750026

12 (2017) 1750142
(2017) 1750109 